\title{
Grating-Coupled Surface-Plasmon-Resonance Disc Biosensor for Monitoring Growth of Self-Assembled Monolayer
}

\author{
Jenq-Nan Yih*, Nai-Jen Cheng1, Kuo-Chi Chiu², \\ Chih-Ming Lin $^{3}$ and Shu-Jen Chen ${ }^{4}$ \\ Department of Electrical Engineering, Kaohsiung University of Applied Sciences, \\ Kaohsiung 807, Taiwan, ROC \\ 'Institute of Photonics and Communications, Kaohsiung University of Applied Sciences, \\ Kaohsiung 807, Taiwan, ROC \\ ${ }^{2}$ Electronics and Optoelectronics Research Laboratories, Industrial Technology Research Institute, \\ Hsinchu 310, Taiwan, ROC \\ ${ }^{3}$ Department of Applied Science, National Taitung University, Taitung 950, Taiwan, ROC \\ ${ }^{4}$ Department of Chemical and Materials Engineering, Kaohsiung University of Applied Sciences, \\ Kaohsiung 807, Taiwan, ROC
}

(Received November 5, 2012; accepted February 4, 2013)

Key words: surface plasmon resonance, disc, fluidic channel, polydimethylsiloxane, selfassembled monolayer

We present a grating-coupled surface plasmon resonance (SPR) disc biosensor for obtaining kinetic information of molecular interaction. The grating disc combined with a c-shape fluidic channel is capable of driving a testing solution forward by gravity by turning the disc biosensor. The disc biosensor can react with probe molecules on a particular sensing surface and then the next as time goes by. To demonstrate the ability of the biosensor, a test experiment was performed for monitoring the growth of a self-assembled monolayer (SAM) immobilized on a gold surface. These measured response curves show that the saturation time of the SAM growth, whose thickness is smaller than $2 \mathrm{~nm}$, is roughly $5 \mathrm{~h}$ when $1 \mathrm{mM}$ 16-mercaptohexadecanoic acid (MHDA)SAM is grown on gold at room temperature. The demonstration reveals that the disc biosensor with c-shape fluidic channels can be a promising tool for a kinetic analysis of bimolecular interaction without any external fluid pumping systems.

"Corresponding author: e-mail: jnyih@kuas.edu.tw 


\section{Introduction}

Label-free optical biosensing technologies are mainly based on surface plasmon resonance (SPR), interferometer, waveguide, fiber Bragg grating, ellipsometry, ring resonators, and photonic crystals. ${ }^{(1)}$ Having the advantages of label-free analyte, high sensitivity, and capability of doing kinetic analysis, SPR biosensors can characterize and quantify biomolecular interactions. ${ }^{(2)}$ SPR is a photoelectric phenomenon, in which the photon energy transfers totally to the surface plasmon under momentum matching condition, and the surface plasmon resonates on the metallic surface without radiating. When the reacting molecules move near the metal surface, the effective dielectric constant of the solution near the surface changes and so does the resonance condition of the SPR. This change can be gauged by the change in the intensity, wavelength, phase or angle of the reflected light. It is by observing these changes that the reacting molecules are detected. Under the momentum matching condition in grating coupling, the parallel component of the wave vector of high-order diffracted light is equal to that of the wave vector of the surface plasma wave. Expressed in mathematics, it is ${ }^{(2,3)}$

$$
\frac{2 \pi}{\lambda_{0}} \sqrt{\varepsilon_{2}} \sin \theta+m \frac{2 \pi}{\Lambda}=\frac{2 \pi}{\lambda_{0}} \sqrt{\frac{\varepsilon_{1} \varepsilon_{2}}{\varepsilon_{1}+\varepsilon_{2}}},
$$

where $\lambda_{0}$ is the wavelength of the incident light in vacuum, $\Lambda$ is the spatial period of the grating, $m$ is the diffraction order, $\varepsilon_{1}$ and $\varepsilon_{2}$ are the dielectric constants of the metal and the dielectric medium, respectively, and $\theta$ is the stipulated incident angle of the probing beam for a specific set of $\Lambda, m, \varepsilon_{1}$, and $\varepsilon_{2}$. It is called the SPR angle $\theta_{\mathrm{SPR}}{ }^{(4)}$

A disc with fluidic channels has the advantage of high throughput screening capability and that the fluid is driven by centrifugal force without the need of external pumping systems. ${ }^{(5-7)}$ Also, an optical disc driven by desktop/notebook computers can be used as a bioassay screening tool. ${ }^{(8)}$ Since it is chemically inert, gold helps examine chemical interactions quantitatively. A compact disc (CD) with a gold reflective layer is suitable for the preparation of a high-quality self-assembled monolayer (SAM) and for performing high-stability electrochemical analysis. ${ }^{(9-11)}$ Furthermore, the grating coated with gold provides opportunities to excite the SPR at the interface of the metal and dielectric. ${ }^{(2,12,13)}$ Grating-coupled SPR discs could be incorporated with a CD-ROM drive to obtain high throughput. ${ }^{(3,14)}$

Here, we proposed a new type of disc system in which the disc is set vertically instead of horizontally as in the other types of similar devices. ${ }^{(5-7,15)}$ Consequently, the sample can be driven by gravitational force rather than by centrifugal force. One merit of this system is that the investigated sample liquid is stationary when we do not turn the disc and thus we can choose some prescribed time to record and observe the state of the biochemical interaction, thus providing the kinetic information of the interaction. Making use of this kind of setup, we have performed preliminary measurements on the SPR angle shifts at the sequentially reacted areas, which reflect the kinetic response of the biomolecular interaction. ${ }^{(4,16)}$ At present, the angular precision of the measurement 
system has been improved by threefold and thus is capable of resolving a smaller response of monolayer growth.

\section{Experiment}

\subsection{Grating design and fluidic channel fabrication}

The software GSOLVER, based on the rigorous vector diffraction, was used to calculate the diffraction efficiency of the reflected and transmitted electromagnetic waves. A gold layer of ca. $50 \mathrm{~nm}$ thickness was coated on the grating substrate to excite SPR and to enhance the field at the metal surface, thus increasing the sensitivity of the biosensor. To reduce the computation time, we used a periodic rectangular profile for the actual geometrical structure of the grating in the simulation. We set the spatial period $\Lambda$ of a periodic rectangular profile as $1.5 \mu \mathrm{m}$, its duty cycle (duty length/ $\Lambda$ ) as 0.40 , and its modulation depth as $64 \mathrm{~nm}$. The parameters were chosen on the basis of the result of atomic force microscopy topography of the actual structure of the grating. Following Dannenberger et al. ${ }^{(17)}$ and Benilova et al., ${ }^{(18)}$ we assumed the thicknesses of SAM as 1.94 $\mathrm{nm}$ and its refractive index as 1.46 at $633 \mathrm{~nm}$ wavelength. The result of the simulated reflectivity at the SPR angle is shown in Fig. 1, in which the shift of the SPR angle corresponding to SAM growth is found to be $0.28^{\circ}$.

Polydimethylsiloxane (PDMS) was adopted to fabricate the fluidic channel because it is inert to biomolecules. Figure 2 shows the cross section of the c-shape fluidic channel. After fabricating the channel mold, we assembled the grating disc, the c-shape fluidic channel mold, and the acrylic cover. The sandwich-assembly scheme is as shown in Fig. 3. After assembly, the grating-coupled SPR disc biosensor combined with the c-shape fluidic channel was obtained.

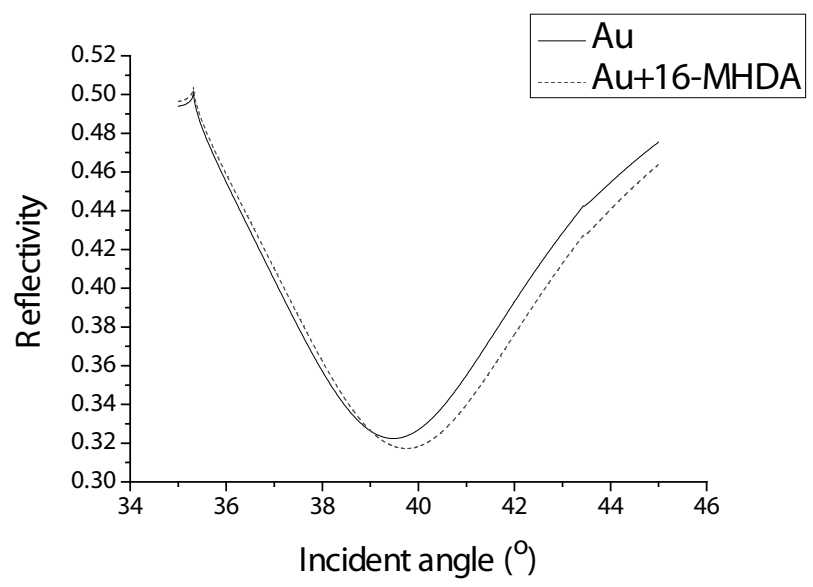

Fig. 1. Curves of simulated reflectivity at SPR angle before and after the growth of an MHDASAM on gold-coated grating. The SPR angle shift is $0.28^{\circ}$. 


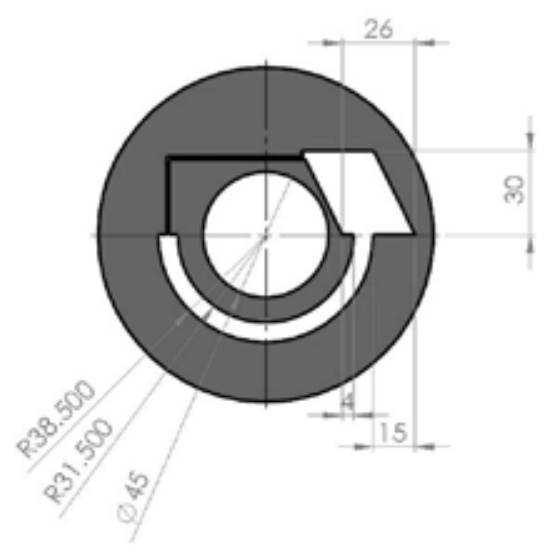

Fig. 2. Cross section of mold of the c-shape fluidic channel, which was made of PDMS (unit: $\mathrm{mm})$.

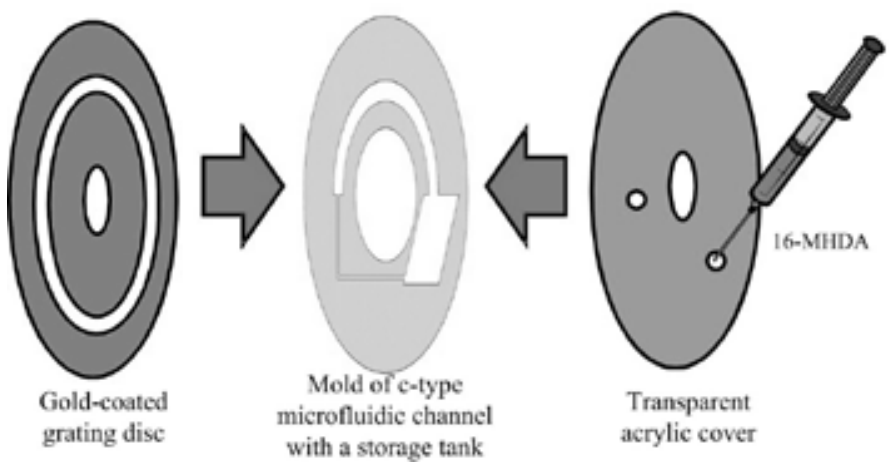

Fig. 3. Biosensor assembly scheme.

\subsection{Metrology}

Our measurement system adopted an architecture similar to that of the corner reflector, so that the system (see refs. 4 and 16) does not need to locate the incident angle and reflection angle simultaneously by synchronously driving two rotators. Instead of making two angular movements as in other setups, the present system can change the incident angle and reflection angle simultaneously just by rotating the shaft of the disc. The SPR angle was obtained from the angle of minimum reflectivity, which resulted from the average of ten measurements with a curve fitting of eighth-degree polynomial.

The result of system repeatability testing is shown in Fig. 4, which shows that the 


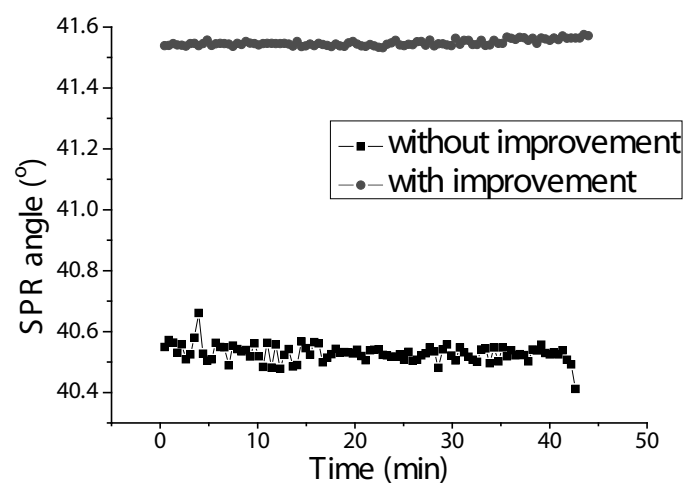

Fig. 4. Result of repeatability test before and after the improvement of system automation. 100 measured points for each set. The angle resolution was improved by threefold from a standard deviation of $0.028^{\circ}$ to $0.0098^{\circ}$ in $40 \mathrm{~min}$.

angle resolution was enhanced by threefold compared with that without adding the automation improvement. ${ }^{(4)}$ An experimental result of SPR measurements before and after a SAM growth is shown in Fig. 5. Two sets of SPR angles were obtained by a series of movements of the sample disc without and with the tested samples, respectively. Then, the SPR angle shifts due to the biomolecular interaction were obtained.

\subsection{Monitoring SAM growth}

SAM is constituted by a tight orderly monolayer of organic molecules. These organic molecules are originally dissolved in solvent and they are spontaneously adsorbed to a substrate by chemical bonding force and the van der Waals force. The thickness of SAM depends on its molecular size and the orientation of arrangement on the surface. Modification of the functional group of SAM is one of the methods often used to capture biomolecules such as protein onto a surface.

16-Mercaptohexadecanoic acid (MHDA, $\mathrm{HS}\left(\mathrm{CH}_{2}\right)_{15} \mathrm{CO}_{2} \mathrm{H}$, Sigma-Aldrich) dissolved in ethanol was used to prepare an MHDA-SAM on gold, which contains two terminated groups: One is a sulfur group -SH that forms a Au-S covalent bond on gold, ${ }^{(19)}$ and the other is a carboxyl acid group - $\mathrm{COOH}$. The growth of MHDA-SAM can be identified by spreading the growth states on the disc along the c-shape channel, and then by measuring the SPR angle shifts on the corresponding locations. Before spreading, the MHDA solution of $1 \mathrm{mM}$ was injected into the storage tank and then the chamber was plugged. Then, the disc stocked with the MHDA solution was put on a rotational stage to perform the spreading of the growth states on the disc along the channel, in which the MHDA solution was moved forward in the c-shape fluidic channel to contact with the sensor surface for $0,1,2,3,4,5,6,12,15,18,24$, and $25 \mathrm{~h}$ in sequence on twelve locations along the c-shape channel by turning the disc. After recording the growth 


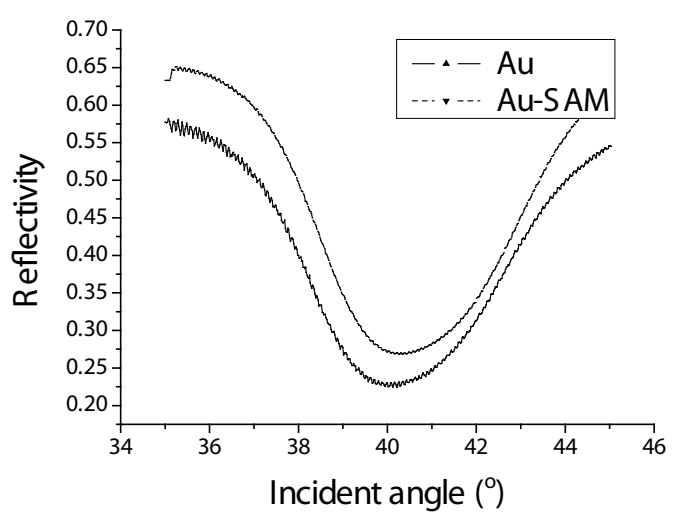

Fig. 5. A set of curves of measured reflectivity at SPR angle before and after MHDA-SAM was grown on the gold-coated grating disc. The SPR angle shift is $0.23^{\circ}$.

states, the MHDA solution was extracted out of the channel by a syringe, and then the channel was rinsed by ethanol injection and dried with pure nitrogen gas. The measured SPR angle shifts corresponding to the growth of MHDA-SAM obtained in this way are shown in Fig. 6, from which the saturation time is found to be around $5 \mathrm{~h}$.

\section{Discussion}

Comparing the simulation (see Fig. 1) and experimental result (see Fig. 5), we found that the fluctuation of reflectivity in Fig. 5 is due to the multilayer interference of the sandwich structure of the disc biosensor. This effect has been considered in the simulation process but the result is not shown in this paper.

Usually, a transient state of biomolecular interaction for kinetic analysis is not recorded on the biosensor for repeating extraction. In the experiment of monitoring the growth of SAM, at specified locations along the c-shape channel on the sensor area, the signal of the molecular interaction state was recorded in the prescribed times. Moreover, if the chemical reaction was fixed on the biosensor, the signal can be repeatedly retrieved even after an irreversible chemical reaction. This data processing method can further reduce the errors due to mechanical action and improve the angular precision.

Two experimental results were obtained for monitoring the growth of MHDA-SAM. Their comparison shows that an obvious difference in response appears in the first 3 h. The result of exp. 2 implies that the SAM has grown on the gold surface gradually as assumed, while that of exp. 1 does not. This inconsistency was found in our study previously, especially at the interaction state in the first $4 \mathrm{~h}$. As far as we know, a sulfursulfur bonding would happen in the self-assembly process of thiols. This interference should be verified and further prevented. However, the above experimental results demonstrated that the disc biosensor is capable of recording the kinetic information 


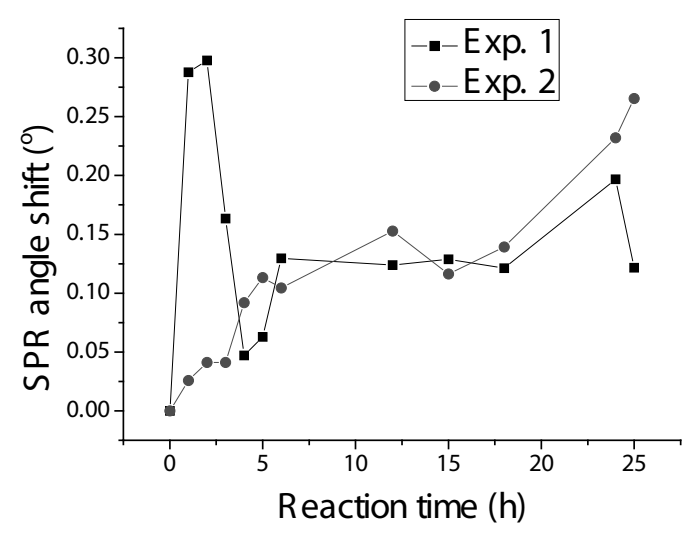

Fig. 6. Measured kinetic response of the growth of MHDA-SAM.

of SAM growth and can identify the thin film growth of a monolayer of only $2 \mathrm{~nm}$ thickness.

\section{Conclusions}

We fabricated a grating-coupled SPR disc biosensor combined with c-shape fluidic channels and a storage tank. The repeatability test shows that the angle resolution was enhanced to $0.044^{\circ}$. An experiment for monitoring the kinetic response of MHDASAM growth was performed. The measured responses showed that the saturation time of the SAM growth was approximately $5 \mathrm{~h}$ when $1 \mathrm{mM}$ MHDA was interacted with the gold surface. These results show that the biosensor has the capability of monitoring the growth of SAM of only $2 \mathrm{~nm}$ thickness. These results support that the proposed gratingcoupled SPR disc biosensor can be a promising tool for kinetic analysis of biomolecular interaction without any external fluid pumping systems.

\section{Acknowledgements}

The authors gratefully acknowledge the support of this study by the Industrial Technology Research Institute of Taiwan.

\section{References}

1 X. D. Fan, I. M. White, S. I. Shopova, H. Y. Zhu, J. D. Suter and Y. Z. Sun: Anal. Chim. Acta $620(2008) 8$.

2 J. Homola, S. S. Yee and G. Gauglitz: Sens. Actuators, B 54 (1999) 3.

3 J. Dostalek, J. Homola and M. Miler: Sens. Actuators, B 107 (2005) 154. 
4 J.-N. Yih, K.-C. Chiu, S.-Y. Chou, C.-M. Lin, Y.-S. Lan, S.-J. Chen and N.-J. Cheng: NEMS IEEE Int. Conf. Nano/Micro Eng. Mol. Syst., (IEEE Computer Society, Piscataway, 2011) p. 602 .

5 M. Madou, J. Zoval, G. Y. Jia, H. Kido, J. Kim and N. Kim: Annu. Rev. Biomed. Eng. 8 (2006) 601.

6 D. D. Nolte: Rev. Sci. Instrum. 80 (2009) 101101.

7 X. Y. Peng, P. C. H. Li, H. Z. Yu, M. Parameswaran and W. L. Chou: Sens. Actuators, B 128 (2007) 64.

8 Y. C. Li, L. M. L. Ou and H. Z. Yu: Anal. Chem. 80 (2008) 8216.

9 L. Angnes, E. M. Richter, M. A. Augelli and G. H. Kume: Anal. Chem. 72 (2000) 5503.

10 H. Z. Yu: Anal. Chem. 73 (2001) 4743.

11 H. Z. Yu: Chem. Commun. 40 (2004) 2633.

12 H. Raether: Surface Plasmons on Smooth and Rough Surfaces and on Gratings (Springer-Verlag, Berlin, 1988).

13 A. D. Boardman: Electromagnetic Surface Modes (Wiley, Chichester, 1982).

14 J. Dostalek and J. Homola: Sens. Actuators, B 129 (2008) 303.

15 L. Wang, P. C. H. Li, H. Z. Yu and A. M. Parameswaran: Anal. Chim. Acta 610 (2008) 97.

16 S.-Y. Chou, W.-Y. Meng, K.-C. Chiu, C.-M. Lin, Y.-S. Lan, N.-J. Cheng and J.-N. Yih: IEEE Int. Conf. Nano/Mol. Med. Eng., NANOMED (IEEE Computer Society, Piscataway, 2009) p. 231.

17 O. Dannenberger, K. Weiss, H. J. Himmel, B. Jager, M. Buck and C. Woll: Thin Solid Films 307 (1997) 183.

18 I. Benilova, V. I. Chegel, Y. V. Ushenin, J. Vidic, A. P. Soldatkin, C. Martelet, E. Pajot and N. Jaffrezic-Renault: Eur. Biophys. J. Biophy. 37 (2008) 807.

19 R. G. Nuzzo and D. L. Allara: J. Am. Chem. Soc. 105 (1983) 4481. 